\title{
Cloud Cover Avoidance in Satellite Systems
}

\author{
https://doi.org/10.3991/ijoe.v16i13.18559 \\ Shreeram Narayanan ${ }^{(凶)}$, Soham Jagtap, \\ Arnold Johnson Fonseca, Reena Sonkusare \\ Sardar Patel Institute of Technology, Mumbai, India \\ shreeram.narayananespit.ac.in
}

\begin{abstract}
Cloud cover is primarily a major difficulty in the acquisition of optical satellite images and has a negative impact on the efficiency of data scheduling. Along with data scheduling, the computational power required is also increasing. Recent advances in an ex-tensive variety of technologies have resulted in an explosion in the amount of data. Different methodologies have been used for Object detection in remote sensing images but it remains a challenge because of its diversity and complex backgrounds. In this paper, a cloud cover detection technique based on Convolutional Neural Networks is proposed for remote sensing images. The classifying model uses a neural network where the underlying features are used to classify the image as useful or not. Results illustrate that the proposed method outperforms other state of the art methods that exist. Once classified, it will be transmitted from the satellite to the earth giving the researchers only convenient pictures to study. This will help to save a massive amount of computation, expense and time.
\end{abstract}

Keywords - Cloud cover, classification, convolutional neural network, satellite images

\section{Introduction}

Necessary world cloud data procured from meteoric satellites needs to be incorporated within the designing system to boost the design potency for maintaining correct knowledge regarding cloud cover. However, the technology is still in a research stage in India and the mechanism is usually implemented with the aid of countries like the United States and Israel; for which the purchasing country has to pay a premium. The sub-sequent presents an analysis of the on the market established technologies in addition as alternative methodologies within the market of our domain.

In 2012, Jiang-Yong proposed a technique for feature extraction and explained various technologies available to carry out training and testing of images to include two forms of set: cloudy category and clear category. The cloudy category possesses pictures beneath completely different cloud conditions, like thick or skinny clouds etc. The clear category co-versa spread of pictures with numerous geographic characters, like ocean, mountain, farmlands and desert [1]. Drawback of this system is that it utilizes the discriminant model for analysis, which is not very efficient for instances where weak classification is pre-sent hence this method is not that viable for analysis. 
In 2013, Xiaofeng Ren [2] suggested a method using computer vision techniques and mathematical modelling. Although, a linear classifier and a simple arbitrary search algorithm have produced hopeful results on a variety of natural images. The downside of this process is that it takes into account the bias that it acquires through the embedded mathematical function and this, in turn, hinders the feature extraction process. In 2017, Zhenwei Shi proposed an algorithm for the purpose of Applied Earth Satellite Observations [3]. How-ever, the algorithm follows a huge range of computer imaging applications, which could then indirectly increase computational time if sufficient hardware power is not available.

In 2017, research by Freeman [4] provides a good risk of implementation of the onboard deep-learning algorithms to unravel the high-resolution remote sensing image classification issues, especially once rough cloud fraction has to be calculated before image transmission. Deep-learning models were further urged to boost the classification facet. From the above analysis it is evident that preprocessing combined with a neural network is an elegant solution which is best for the use of the remote sensing application. This paper presents such methodology to help classify the cloud cover in an efficient manner.

\section{Objectives}

In satellite obtained images, presence of obstruction in the form of clouds affects processing of images and subsequent data quality, as the images having clouds comprise of diminutive valuable information. Hence, detecting such an obstruction is a big problem in the function of these satellite pictures. At the moment, two methods exist for cloud determination. One is to adjust band thresholds on the basis of features of the clouds to differentiate them. Yet, the variability and vagueness of clouds in practice make such a method com-plex and frail in terms of adaptability and the incentives are:

- Data quality, accurate area of coverage (targeting accuracy), promptness and cloud free acquisition are parameters which are of importance for the prime use of satellite re-sources to satiate user demand.

- Improvement in performance, efficiency and cost. Currently, the chance of mis-judgment for the cloud detection methodology features is comparatively high.

- Predicting cloud cover depending on the previously interpreted scheduling time. This will hence focus on a database of mean seasonal cloud cover for every area of interest.

A comparison with completely different algorithms as well as machine learning based methodology and threshold-based methodology on the same data-set suggests effectiveness of deep learning models and proves the validity and potency of our algorithmic rule. 


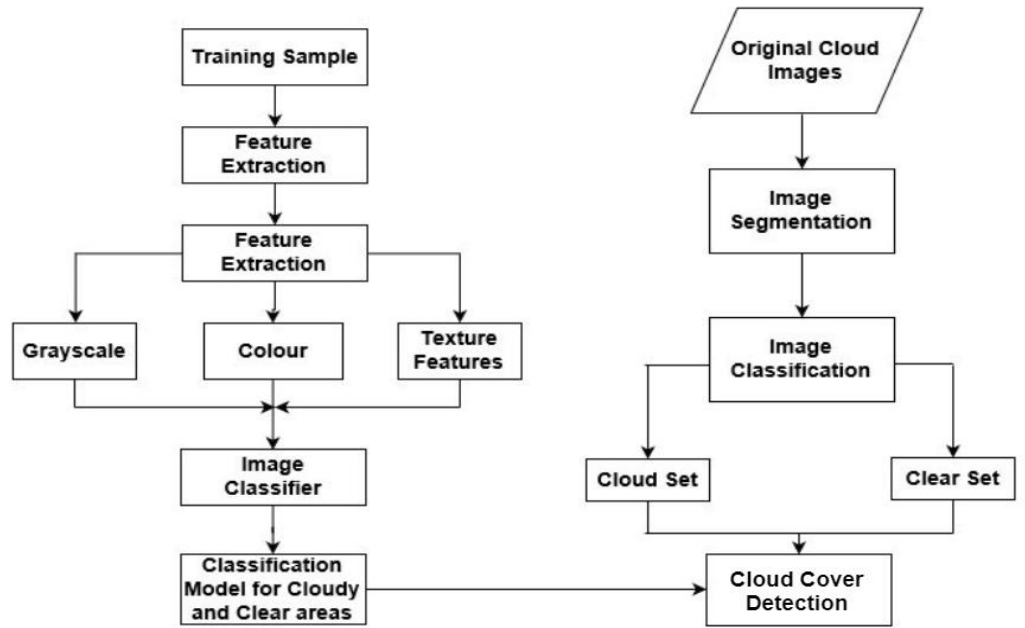

Fig. 1. Flowchart

\section{Proposed System and Design}

The design of this application is to handle the inefficiency of current systems in terms of portability and hardware compatibility. Hardware Compatibility is associated with resource handiness and capability of storage for the remote sensing images. Under-performance of alternative segmentation algorithms along-side normalized overcast prediction because of either serious compression presence of weak classifiers are some of the drawbacks the instructed answer focuses to handle. A large infrastructure exists among the sphere of image databases, image processing, human portable computer interfaces and image visualisation. Add to that, a thorough understanding of the metrics, familiarity with the description and explaining footage is pivotal to the application of this project. The varied capabilities of satellites and its resources demand a meticulous resolution to come back up with.

The entire process focuses on the extraction of features from the data-set, following which it will aid in the purpose of image classification. As illustrated in the flowchart in Fig 1, the training sample is utilized for feature extraction and the classification model will be centered primarily around three attributes. They will be Grayscale, Colour and Texture features respectively, which will be embedded into the classification model. Likewise, from the original data-set of cloud cover at hand, image segmentation is performed using processing algorithms and it is then classified into a cloudy dataset and clear dataset based on a certain level of threshold [5]. Whilst Employing the classification model for determining clear and cloudy skies via neural network, the images will also be classified as having a considerable cloud cover or not.

Hence, the functioning is a multistage process and also integrated in such a way that it is initialized by the segmentation module during pre-processing. Lastly, this information is sent through the neural network and the output is obtained. This Results in an 
elegant solution for the classification problem. All The photographs can be visualized via Remote Pixel Viewer, that provides a listing of default band combinations, still as further image intensification tools aboard many alternative base-maps. The current CNN could be a supervised machine learning approach, and so needs many labelled pictures for the training part. For Imagery: Remote Pixel features only 3 satellite imagery data-sets: Landsat 8, Sentinel-2, and CBERS-4. Image Catalog features [6] nearly a dozen satellite image collections for land cover, water resources monitoring, vegetation, as well as meteorological observations. One of its benefits is the ability to research and visualize image information. The images from all satellites were assembled and labelled, creating a data-set for image analysis and classification purpose. The images with noise (irrelevant images with respect to the data-set), should be re-moved. Hence, detecting regions having cloud cover are vital to enhance the usefulness of remote sensing data.

\section{$4 \quad$ Methodology}

We have created a new data-set for cloud detection purposes and adopted a method based on Convolutional Neural Networks (CNN) for satellite sensing data. We then trained our system and inspected the validation set as in [7]. It is used to perform particular operations on the image and to extract useful information about the presence of cloud from it. The Method consists of the following components along with their functionalities: For the process of classification of images from the data-set, a Keras sequential model was created by passing a list of layer instances. An argument input_shape was passed to the first convolutional layer. Maximum pooling method was used after adding the pooling layer assisted by:

- Compilation: [6] The classifier compile function consists of three arguments; an optimizer, a loss function and metric, leading to execution in terms of epochs.

- Training: [7] The training of the model was done using the classifier function, iterating on the data in batches of 128 samples. We set different parameter levels to obtain different values of accuracy.

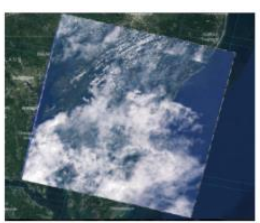

(a) Clouded

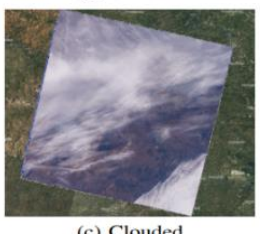

(c) Clouded

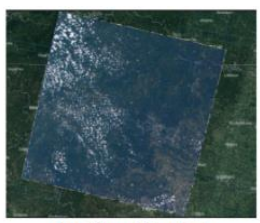

(b) Non-Clouded

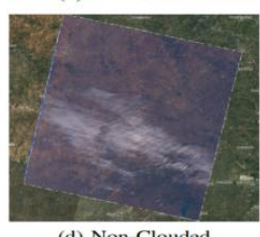

(d) Non-Clouded

Fig. 2. Classification for Training 


\subsection{The convolutional neural network}

The concept of Artificial intelligence for images was introduced almost two decades back but the efficiency of the models proposed was quite less compared to the prevalent technology using image processing for analysis. The inefficiency existed due to poor models that were proposed and also less flexibility on the computation front. But the improvements in the computational power and the introduction of robust Neural Networks has led to an in-creasing preference of deep-learning models over conventional image processing. With substantial results and progress shown by neural networks for processing computer and robotic vision problem statements, they are used immensely in classification of image and as well as it's segmentation. CNNs can automatically learn both low-level and high-level features. Here, we extract the cloud features and classify clouds from land covers based on CNN.

Instinctively, the situation of the feature in accordance with the opposite options is vital instead of the real location of the feature itself. Pooling is done for reducing the dimensions of the input so that it retains only required details and makes computation less expensive. The hidden multiple neural layers are used to extract the underlying nonlinear features from the input. The collected pictures were cropped to squares and labelled as shown in Fig. 2. Labelling is done for the training purpose of CNN. The pictures additionally contain numerous geographical and topographical options that result in standardization. The adjusted coaching data-set hada homogeneous probability for every cloud class, when the implementation of the standardization procedure.

In distinction, we have a tendency to use a unique version of a CNN design for image seg-mentation to classify a whole image in an all-inclusive manner [8]. The Convolutional Network used is precise in classifying the clouded and non-clouded images and at the same time avoiding the computation of unnecessary data.

\subsection{Dropout for deep neural networks}

Deep Neural Networks comprises multiple nonlinear hidden layers that enables them to learn sophisticated patterns between the inputs and outputs. However, over-fitting may be a significant issue in such networks wherever the model performs adequately on the coaching information however does not perform well on unseen test information. To solve the problem of overfitting many regularization methods have been proposed like weight decay, L1, L2 regularization, data augmentation etc. Dropout empirically proven to be more efficient. Dropout regularization technique was used to dropout hidden units in the neural network [9]. The probability of retention was set as 0.5 for the hidden layers which seems to work well for a wide range of networks and applications.

\section{$5 \quad$ Evaluation and Results}

The deep-learning model was tested by utilizing different optimizing functions along with learning rates and epochs. Best model was selected on observing the final accuracy of the training and validation set discussed in [10]. An effective on-board cloud 
detection methodology in little satellites would greatly improve the down-link knowledge transmission potency and cut back the memory price.

The model that we have proposed consists of a classifier built with 4 hidden layers with the number of neurons in each layer varying between 32 and 64 . When the neurons of the layers other than input and output had an activation of ReLU. The output classifying neuron, however, gave the best result with sigmoid activation.

The convolutional layer extracts feature from pictures with filters that an area unit applies to the initial image within the model. The Associate degree Activation map of that filter is made with the assistance of the activation function applied. In the pooling layers, what happens could be a form of down sampling. A subsequent convolution layer will then learn to assemble an additional precise output supporting this data. The model was over-fitting with a high training ac-curacy of about $96 \%$ and a validation accuracy of about $87 \%$. To solve the problem of high variance which happens when a model over-fits, regularization techniques were applied. The addition of dropout layers after the fully connected layers helped solve this problem and improve validation accuracy.

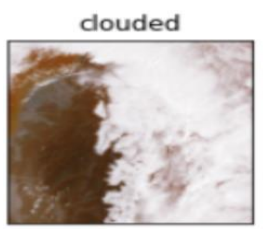

clouded

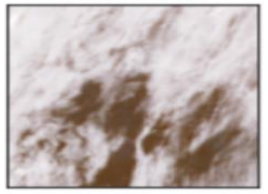

Fig. 3. Classification by Model

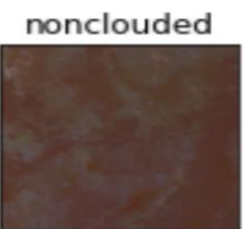

nonclouded

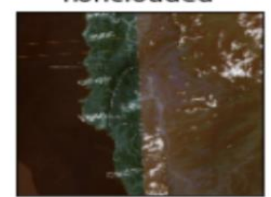

Adam optimizer gave us the best result with binary cross entropy as the loss function. The problem of overfitting was solved using regularization techniques and the model performed very well on new test sets. The overall accuracy of different algorithms is indicated in Table 1. The impact of the dropout layer is that the network becomes less receptive to the specific weights of neurons. This successively ends up in a network that is able to generalize and thus re-duces the possibility of over-fitting the training set. The highest accuracy was $92.5228 \%$ as shown in Table 1 .

The model was then compiled and highly accurate results in relation to validation set were detected as per Fig. 3. Thus, training a convolutional neural network such as this utilizes a supervised learning method with a properly labelled classification. The results obtained show that good levels of accuracy are attained. A comparison between all the optimizers used with respect to training and testing accuracy is given in Fig. 4. 
Table 1. Validation Set Statistics

\begin{tabular}{|l|c|c|}
\hline \multicolumn{1}{|c|}{ Optimizer } & Epochs & Accuracy (\%) \\
\hline \multirow{3}{*}{ ADAM (with dropout) } & 30 & 92.5228 \\
\cline { 2 - 3 } & 10 & 85.6718 \\
\cline { 2 - 3 } & 15 & 85.8725 \\
\cline { 2 - 3 } & 20 & 87.2576 \\
\hline SGD & 30 & 83.3379 \\
\hline SGD & 10 & 49.3074 \\
\hline RMSPROP & 20 & 50.7678 \\
\hline
\end{tabular}

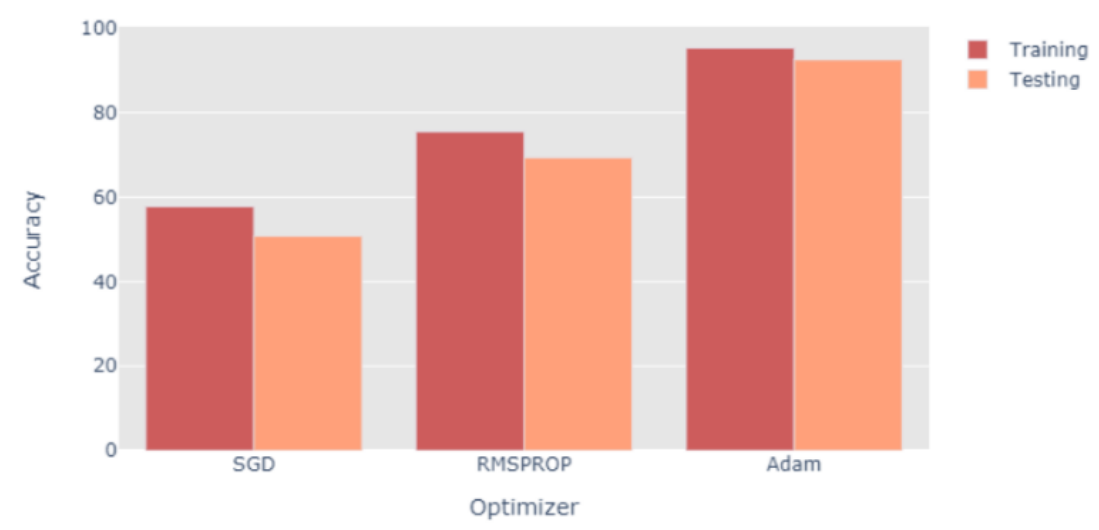

Fig. 4. Comparison of Optimizer Performance

Qualitative analysis is also enforced within the experimental stage. The validation set comprised $20 \%$ of the data. The accuracy plot demonstrated in the Fig. 5 indicates the performance of the previous model implemented. The loss performance too, is given in Fig. 6. Although the model validation accuracy is adequately high but the loss parameters are not satisfactory and hence this model is inefficient.

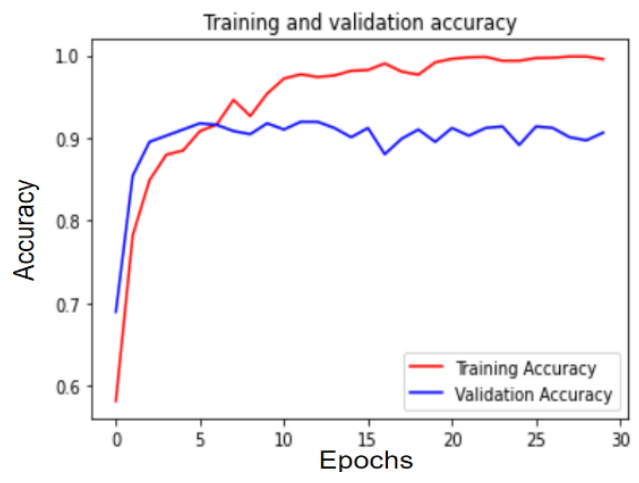

Fig. 5. Accuracy Plot 


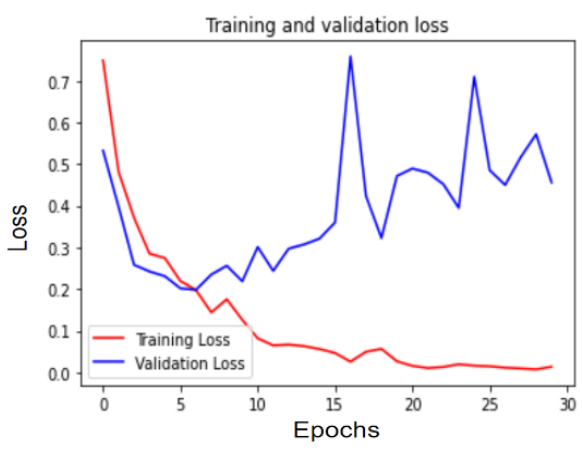

Fig. 6. Loss Parameters

The considerable improvement validation accuracy using drop-out layers is given by observing the plots illustrated in Fig. 7. The Loss parameters also display a wellgroomed convergence factor which is better in terms of loss performance as shown in Fig. 8 .

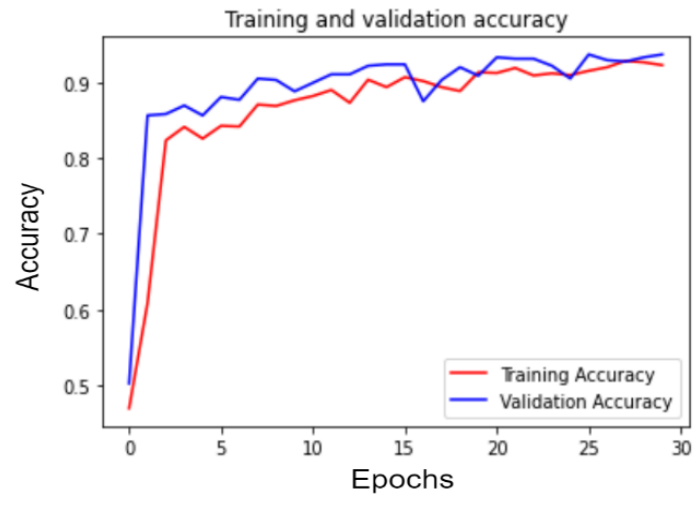

Fig. 7. Accuracy Plot

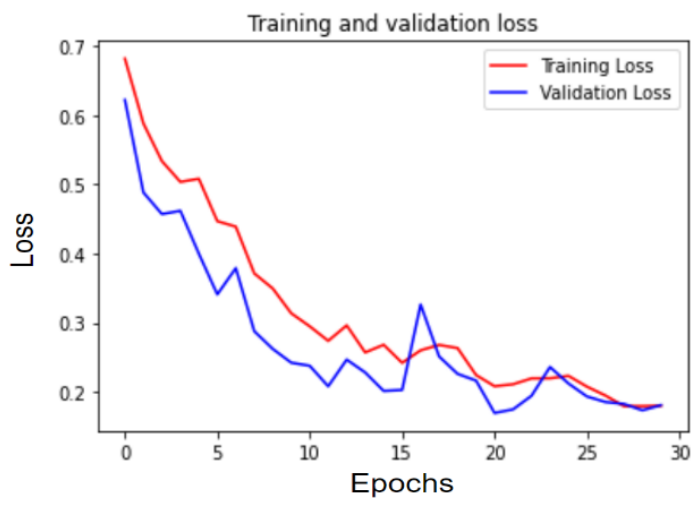

Fig. 8. Loss Parameters 


\section{Conclusion}

Recent advances in a wide selection of technologies have led to an explosion in the quantity as well as quality of satellite image classification. The requisite CNN methodology proves efficient in cloud cover detection for several satellite images. Experimental results show that classification using $\mathrm{CNN}$ gives a higher accuracy compared to other existing methods for image classification. The urged classification technique on remote sensing might be widely applied to varied applications like military target recognition, environment observation, meteorology, mineral development and geographical mapping. By taking advantage of the system in satellites, the planned technique provides a decent possibility of on-board cloud detection supported by deep learning.

\section{$7 \quad$ References}

[1] CHEN, Wen-jia, Jiang-yong DUAN, and M. E. N. G. Juan. "Cloud detection via convolutional neural network in visible light remote sensing images." DEStech Transactions on Computer Science and Engineering aita (2017). https://doi.org/10.12783/dtcse/aita2017/ $\underline{15987}$

[2] Liu, Bingwei, et al. "An adaptive process-based cloud infrastructure for space situational awareness ap-plications." Sensors and Systems for Space Applications VII. Vol. 9085. International Society for Op-tics and Photonics, 2014. https://doi.org/10.1117/12.2053759

[3] He, Lei, et al. "Cloud Avoidance Scheduling Algorithm for Agile Optical Satellites." Journal of Computational and Theoretical Nanoscience 13.6 (2016): 3691-3705. https://doi.org/ 10.1166/jetn.2016.5201

[4] Henry TR, Kilpatrick FA, Skinner JM. Space image acquisition and processing architecture. In1998 IEEE Aerospace Conference Proceedings (Cat. No. 98TH8339) 1998 Mar 28 (Vol. 2, pp. 165-172). IEEE. https://doi.org/10.1109/aero.1998.687908

[5] Stoll E, Schulze R, D'Souza B, Oxfort M. The impact of collision avoidance maneuvers on satellite con-stellation management. Inproc. Of European Space Surveillance Conference, Madrid, Spain 2011 Jun.

[6] Xiu H, Yang F. Batch Processing of Remote Sensing Image Mosaic based on Python. International Journal of Online and Biomedical Engineering (iJOE). 2018 Sep 30;14(09): 208-16. https://doi.org/10.3991/ijoe.v14i09.9226

[7] Gasch JR, Campana KA. Cloud cover avoidance in space-based remote sensing acquisition. InAlgo-rithms for multispectral, hyperspectral, and ultraspectral imagery VI 2000 Aug 23 (Vol. 4049, pp. 336-347). International Society for Optics and Photonics. https:// doi.org/10.1117/12.410357

[8] Ouassit Y, Ardchir S, Moulouki R, El Ghoumari MY, Azouazi M. Liver Segmentation A Weakly End-to-End Supervised Model. International Journal of Online \& Biomedical Engineering. 2020 Sep 1;16(9). https://doi.org/10.3991/ijoe.v16i09.15159

[9] Zeng Y, Lan J, Han C, Jiang L, Shi X. Hyperspectral Remote Sensing Data Recovery Via Adaptive Window Matching Method. International Journal of Online and Biomedical Engineering (iJOE). 2015 Jan 25;11(1):39-45. https://doi.org/10.3991/ijoe.v11i1.4235

[10] Changhui Y, Yuan Y, Minjing M, Menglu Z. Cloud detection method based on feature extraction in re-mote sensing images. International Archives of the Photogrammetry, Remote Sensing and Spatial In-formation Sciences. 2013 2: W1. https://doi.org/10.5194/isprsarchives-xl-2-w1-173-2013 


\section{Authors}

Shreeram Narayanan is a student in Electronics and Telecommunication Engineering at Sardar Patel Institute of Technology, Mumbai. Email: shreeram.narayanan@spit.ac.in

Soham Jagtap is a student in Electronics and Telecommunication Engineering at Sardar Patel Institute of Technology, Mumbai. Email: soham.jagtap@ spit.ac.in

Arnold Johnson Fonseca is a student in Electronics and Telecommunication Engineering at Sardar Patel Institute of Technology, Mumbai. Email: arnoldjohnson.fonseca@spit.ac.in

Dr. Reena Sonkusare is a Professor and Head of Electronics and Telecommunication Engi-neering Department at Sardar Patel Institute of Technology, Mumbai. Email: reena_kumbhare@spit.ac.in

Article submitted 2020-09-14. Resubmitted 2020-10-13. Final acceptance 2020-10-17. Final version published as submitted by the authors. 